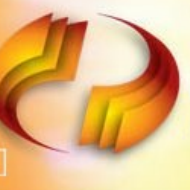 PRDDUCFĀ ONLINE REVISTA CIENTIFICA ELETRÔNICA DE ENGENHARIA DE PRODUÇÃO ISSN 1676-1901
}

\section{QUALIDADE DA INFORMAÇÃO NO PCP: ANÁLISE DOS FATORES DE INFLUÊNCIA E PROPOSTA DE UM MÉTODO DE DIAGNÓSTICO}

\section{INFORMATION QUALITY FOR PPC: ANALYSIS OF INFLUENCE FACTORS AND DIAGNOSIS METHOD PROPOSAL}

\author{
Eduardo Belmonte Möller* E-mail: eduardobmoller@gmail.com \\ Guilherme de Oliveira Schattschneider* E-mail: g.schatts@gmail.com \\ Alejandro Germán Frank* E-mail: agerfrank@producao.ufrgs.br \\ José Luis Duarte Ribeiro* E-mail: ribeiro@producao.ufrgs.br \\ *Universidade Federal do Rio Grande do Sul (UFRGS), Porto Alegre, RS
}

\begin{abstract}
Resumo: Um dos principais problemas que as empresas devem enfrentar no Planejamento e Controle da Produção (PCP) é a disponibilidade de qualidade da informação (QI) para subsidiar a tomada de decisões. Neste contexto, uma questão-chave é determinar quais os fatores que prejudicam a QI necessária para o PCP. Assim sendo, este artigo apresenta um estudo de caso no qual se realiza um levantamento dos principais fatores que influenciam na QI para o PCP. Além disso, propõe-se um método para a realização do diagnóstico da situação desses fatores nas empresas. Para analisar os fatores da QI foi utilizada uma classificação baseada na abordagem de sistemas sociotécnicos e foram utilizadas matrizes de relacionamentos entre fatores e áreas funcionais da empresa. Os resultados permitem identificar quais os principais fatores, áreas e processos que apresentam oportunidades de melhoria no que concerne à QI para o PCP. O trabalho apresenta resultados práticos que ilustram a forma de conduzir diagnósticos da QI no PCP.
\end{abstract}

Palavras-chave: Qualidade da informação (QI). Planejamento e Controle da Produção (PCP). Método de diagnóstico. Estudo de caso.

\begin{abstract}
One of the most important problems that companies have to face for Production Planning and Control (PPC) is the lack of Information Quality (IQ) to support the decision making process. In this context, determining which factors affect the IQ necessary for PPC is a key issue. This paper presents a case study that analyzes the main influence factors on IQ. Furthermore, this paper proposes a method to perform diagnosis about these influence factors in companies. To analyze the IQ influence factors, a classification based on the sociotechnical approach was used and a set of matrixes that relate the IQ influence factors with the different functional areas that support the PPC were proposed. Results enable to identify which are the main factors and the functional areas and processes that can be improved to increase the IQ for PPC. This paper presents also practical results that show how to drive an IQ diagnosis.
\end{abstract}

Keywords: Information Quality (IQ). Production Planning and Control (PPC). Diagnosis method. Case study.

\section{INTRODUÇÃO}

A competitividade empresarial depende em larga escala do desempenho dos seus processos internos. Nesses processos, realizam-se continuamente decisões de cunho gerencial e operacional, que estão baseadas em informações geradas e disponibilizadas por diferentes setores dentro da 
empresa. Conforme afirma Moresi (2000), a informação é um recurso-chave, cuja gestão e aproveitamento estão diretamente relacionados com o sucesso nas tomadas de decisões. Portanto, precisa-se que estas informações sejam disponibilizadas no momento certo e para a finalidade certa.

Nesse contexto, os Sistemas de Informações (SI) têm uma importância destacada (LAUDON e LAUDON, 2004; DAVENPORT e PRUSAK, 1998).

Contudo, dispor SI para o gerenciamento das informações nem sempre garante que os solicitantes recebam as informações necessárias. Davenport e Prusak (1998) destacam que, muitas empresas, se preocupam em possuírem sistemas sofisticados para a gestão de suas informações, mas não se preocupam em assegurar que as informações disponibilizadas sejam relevantes e atendam às necessidades dos processos. Informações de baixa qualidade podem comprometer os resultados de uma empresa, uma vez que as decisões tomadas são baseadas nessas informações (FAVARETTO e VIEIRA, 2007). A baixa Qualidade da Informação (QI) pode ter um importante impacto negativo na efetividade geral de uma organização (WAND e WANG, 1996). Além disso, organizações que não sabem como tratar as informações que possuem podem estar desperdiçando um valioso recurso estratégico que poderia ser usado para ganhar vantagem competitiva no mercado (KAHN et al., 2002).

Um dos processos nos quais a QI é chave é no Planejamento e Controle da Produção (PCP), pois os resultados finais da empresa são diretamente vinculados à capacidade produtiva que a mesma possui. A QI utilizada para o PCP define a capacidade de planejamento industrial e, portanto, a capacidade de atender às necessidades do mercado, que impacta nos resultados econômicos da empresa (DUTRA e ERDMANN, 2006). O PCP é um dos processos mais complexos nas empresas, devido ao grande número de variáveis que influenciam no sistema e da interdependência entre as mesmas e fatores externos (KLIPPEL et al., 2005). A complexidade cresce quando as informações recebidas não são confiáveis.

Em razão disso, o presente trabalho tem por objetivo realizar um estudo sobre a QI utilizada no processo de PCP, analisando quais os principais fatores que influenciam na capacidade de fornecer informações e propondo uma estrutura que facilite a análise de diagnóstico do impacto da QI no PCP. O artigo apresenta um estudo de caso conduzido em uma empresa metal-mecânica, na qual se realizou o levantamento dos fatores que prejudicam a QI e se desenvolveu uma ferramenta par avaliar a importância da QI no caso analisado. A análise dos fatores é baseada na abordagem de sistemas sociotécnicos, que considera aspectos humanos, organizacionais, sociais e tecnológicos. A principal contribuição deste trabalho é apresentar problemas específicos levantados 
em um caso prático, através dos quais foi possível estudar os fatores de influência sobre a QI. Com isto pretende-se fornecer direcionamentos que ajudem a outras empresas realizarem diagnósticos da QI utilizada no PCP, podendo-se, assim, desenvolver ações de melhoria para os processos envolvidos na criação, disseminação e uso das informações.

A apresentação do trabalho está dividida em três partes. A primeira parte é composta pelos conceitos teóricos que fundamentam a pesquisa. Na segunda parte do trabalho apresentam-se as etapas do método de pesquisa. A última parte contempla a apresentação dos resultados, as discussões desses resultados, as conclusões e as sugestões para trabalhos futuros.

\section{QUALIDADE DA INFORMAÇÃO}

A literatura revela diferentes abordagens para tratar o tema QI. Wang e Strong (1996), por exemplo, utilizam em seus estudos sobre QI o conceito de adequação ao uso como definição de qualidade. Eppler (2003) considera duas dimensões da QI: atendimento às expectativas dos usuários e atendimento às demandas do processo. Redman (1996) define uma informação de qualidade como uma informação livre de defeitos e que possua as características desejadas. Stvilia et al. (2007) ressaltam que a Q é contextual e deve ser analisada considerando o ambiente de estudo. Já Ge e Helfert (2007) vão além e afirmam que uma mesma informação pode ser de qualidade ou não dependendo do contexto em que é analisada, da pessoa que faz a análise ou mesmo da especificação em questão. Assim sendo, não existe consenso sobre definições teóricas e práticas para QI (SORDI et al., 2008). Entretanto, conforme Ge e Helfert (2007), a maior parte dos estudos sobre o tema tenta responder a três questões principais: (i) Como medir a QI?; (ii) Como gerenciar a QI?; (iii) Como a QI impacta no contexto organizacional?

A QI recebeu maior atenção a partir de 1989, quando, no Seminário do Nordic Council for Scientific Information and Research Libraries - NORDINFO, Marchand (1989) apresentou cinco abordagens utilizadas para definição do conceito de QI (Figura 1). Estas cinco abordagens são derivadas de uma proposta anterior realizada por Garvin (1984) para a qualidade dos produtos. Marchand (1989) aplicou a proposta de Garvin (1984) ao contexto da informação, considerando a mesma também como um "produto" que deve possuir qualidade para os clientes que a utilizam. 
Figura 1 - As cinco abordagens de QI segundo Marchand (1989)

\begin{tabular}{|l|l|}
\hline \multicolumn{1}{|c|}{ Abordagens } & \multicolumn{1}{c|}{ Conceptualização } \\
\hline Transcendente & $\begin{array}{l}\text { O valor da informação é absoluto e universalmente } \\
\text { reconhecido, ou seja, a qualidade é intrínseca. }\end{array}$ \\
\hline Baseada no usuário & $\begin{array}{l}\text { O valor da informação está relacionado ao quanto ela } \\
\text { satisfaz as particularidades individuais de seus } \\
\text { usuários. }\end{array}$ \\
\hline Baseada no produto & $\begin{array}{l}\text { Mede a Ql através de atributos precisos e } \\
\text { identificáveis, passíveis de serem mensurados e } \\
\text { quantificados. }\end{array}$ \\
\hline Baseada na produção & $\begin{array}{l}\text { Mede a Ql através de o quanto ela satisfaz aos } \\
\text { padrões estabelecidos de necessidades de seus } \\
\text { usuários. }\end{array}$ \\
\hline Baseada no valor & $\begin{array}{l}\text { O valor da informação é uma categoria mais } \\
\text { abrangente, e sua qualidade um de seus atributos. }\end{array}$ \\
\hline
\end{tabular}

Após os primeiros estudos sobre QI realizados por Marchand (1989), Wang e Strong (1996) conduziram uma pesquisa empírica com o propósito de identificar as dimensões de qualidade de dados e a importância de cada atributo na percepção dos consumidores, identificando categorias e dimensões para qualidade da informação com base na visão do usuário e em critérios semânticos. Posteriormente, Huang, Lee e Wang (1999) realizaram um estudo de caso em projetos de diferentes organizações e adaptaram as dimensões da qualidade de informação listadas por Wang e Strong (1996), obtendo-se como resultado as quatro categorias da informação apresentadas na Figura 2 , bem como suas respectivas dimensões.

Figura 2 - Categorias e dimensões de qualidade de informação

\begin{tabular}{|l|l|}
\hline \multicolumn{1}{|c|}{$\begin{array}{c}\text { Categorias da } \\
\text { Informação }\end{array}$} & Dimensões da Qualidade da Informação \\
\hline \multirow{3}{*}{ Intrínseca } & Com acurácia \\
& Objetiva \\
& Com credibilidade \\
& Fidedigna \\
\hline & Relevante \\
& Com valor agregado \\
& Atualizada \\
& Completa \\
& Com valor apropriado \\
\hline \multirow{3}{*}{ Representacional } & Interpretável \\
& De fácil entendimento \\
& Com representação concisa \\
& Com representação consistente \\
\hline \multirow{2}{*}{ Acessibilidade } & Acessível \\
& Segura \\
\hline
\end{tabular}


Outro trabalho que classifica a QI em diferentes níveis e categorias foi apresentado por Lee et al. (2002). Estes autores desenvolveram um modelo para avaliação da qualidade de informação nas empresas. Tal modelo, chamado AIMQ (Methodology for Information Quality Assessment), classifica as dimensões da qualidade de informação em quatro classes, conforme a Figura 3. Além disso, podem-se acrescentar os critérios de classificação da QI propostos por Naumann e Rolker (2000): critérios subjetivos, objetivos e critérios de processo. Esses critérios ainda podem ser classificados conforme: a percepção do usuário, a informação com valor em si mesma e a acessibilidade da informação (NAUMANN e ROLKER, 2000).

Figura 3 - Classes e dimensões da qualidade de informação

\begin{tabular}{|c|c|c|}
\hline & $\begin{array}{l}\text { Conformidade com as } \\
\text { especificações }\end{array}$ & $\begin{array}{c}\text { Atende ou excede as expectativas do } \\
\text { consumidor }\end{array}$ \\
\hline \multirow{2}{*}{$\begin{array}{l}\text { Qualidades } \\
\text { da } \\
\text { informação } \\
\text { como } \\
\text { produto }\end{array}$} & $\begin{array}{l}\text { Características da informação } \\
\text { atendem aos padrões de qualidade } \\
\text { da informação. }\end{array}$ & $\begin{array}{l}\text { Informação atende às necessidades de } \\
\text { trabalho do consumidor }\end{array}$ \\
\hline & $\begin{array}{l}\text { Livre de erro } \\
\text { Com representação concisa } \\
\text { Completa } \\
\text { Com representação consistente }\end{array}$ & $\begin{array}{l}\text { Quantidade apropriada } \\
\text { Relevante } \\
\text { Compreensível } \\
\text { Interpretável } \\
\text { Objetiva }\end{array}$ \\
\hline \multirow{2}{*}{$\begin{array}{l}\text { Qualidades } \\
\text { da } \\
\text { informação } \\
\text { como serviço }\end{array}$} & $\begin{array}{l}\text { Processo de conversão de dados } \\
\text { em informações atende aos padrões }\end{array}$ & $\begin{array}{l}\text { Processo de conversão de dados em } \\
\text { informações excede às expectativas dos } \\
\text { consumidores }\end{array}$ \\
\hline & $\begin{array}{l}\text { Atualizada } \\
\text { Segura }\end{array}$ & $\begin{array}{l}\text { Com credibilidade } \\
\text { Acessível } \\
\text { Fácil de manipular } \\
\text { Fidedigna } \\
\text { Com valor agregado }\end{array}$ \\
\hline
\end{tabular}

Dentre as dimensões da qualidade observadas pelos diferentes estudos, alguns autores priorizam umas em detrimento de outras, não havendo um consenso de quais possuem maior importância. Sherman et al. (2005), por exemplo, comentam que a informação deve poder ser acessada de forma rápida e eficiente, caso contrário, sua qualidade é comprometida. Os autores destacam a importância de um sistema de informação bem organizado, que permita o acesso à informação de forma eficaz. Por outro lado, Favaretto e Vieira (2007) priorizam as dimensões acuracidade, credibilidade e temporalidade, pois julgam que são as que mais influenciam no processo de planejamento da produção. Já Paim et al. (1996) citam a valorização dos atributos atualidade, validade e confiabilidade em estudos anteriores.

Embora existam diversas tentativas para avaliar e medir a QI, com diferentes enfoques, alguns conceitos fundamentais foram consolidando-se ao longo dos trabalhos. Dentre eles, observa- 
se uma valorização da avaliação da QI conforme percepção do usuário, a avaliação da qualidade de informação dentro do contexto em que ela é utilizada e a avaliação da qualidade de informação como fator de influência nos objetivos das organizações. Porém, todos os fatores da Q apresentados nesta seção também dependem do contexto na qual esta é estudada. Um dos contextos nos quais a QI é relevante é no Planejamento e Controle da Produção (PCP), alvo de estudo do presente trabalho. Nesse contexto, existem vários aspectos que influenciam na QI, que podem ser organizados como fatores sociais, organizacionais e técnicos. Assim sendo, o estudo aprofunda a QI no PCP focalizando nesses diferentes fatores, como é descrito a seguir.

\subsection{Informações utilizadas para o Planejamento e Controle da Produção (PCP)}

O Planejamento e Controle da Produção (PCP) podem ser entendidos como uma área funcional ou, também, como um processo das empresas. Na visão de processos, entende-se o PCP como o processo responsável pela transformação da matéria prima em produto acabado, otimizando o fluxo de materiais e o uso de recursos, reduzindo estoques intermediários e lead times (CIURANA et al., 2008). Trata-se de uma atividade de apoio gerencial que planeja e controla as atividades de manufatura. Zaccarelli (1979) define as atividades de PCP como um conjunto de funções interrelacionadas que objetivam comandar o processo produtivo e coordená-lo com os demais setores administrativos da empresa. No PCP são recebidas informações sobre estoques existentes, vendas previstas, linha de produtos, modo de produzir, capacidade produtiva, etc. A transformação dessas informações em ordens de fabricação é o objetivo deste processo (DUTRA e ERDMANN, 2006).

Para a execução do planejamento de produção é realizado um conjunto de atividades que, sequenciadas e interligadas, permitem que o objetivo principal seja alcançado. Mula et al. (2006) define quatro funções típicas de planejamento da produção: gerenciamento da demanda, planejamento das necessidades de material, planejamento da capacidade e programação e sequenciamento de tarefas. Essas funções são frequentemente gerenciadas por meio de um Sistema de Informação (SI). Neste sentido, os sistemas mais abrangentes são os ERP (Enterprise Resource Planning - Planejamento de Recursos Empresariais). O ERP realiza a conexão de processos e sistemas das diferentes áreas das organizações, formando um sistema altamente integrado que possibilita o compartilhamento de informações por toda a empresa (XU et al., 2006). Neste tipo de sistemas, as informações são coletadas de diferentes fontes para serem fornecidas aos seus respectivos usuários, que podem ser considerados clientes da informação. As informações 
transmitidas ao PCP para realização de suas atividades estão inseridas em ambientes que, por vezes, comprometem sua QI. É por isso que o presente trabalho propõe estudar os fatores de influência na QI existentes neste ambiente de maneira que se possa melhorar o desempenho do PCP.

\subsection{Pesquisas existentes sobre o tema}

A QI é um tema que tem despertado o interesse de vários pesquisadores. A literatura que trata sobre este tema é ampla e a maior parte da mesma concentra-se no estudo dos atributos da QI. Por exemplo, Oleto (2006) estudou a percepção da QI pelos usuários, conforme os diferentes atributos que definem a QI. Sordi et al. (2008) estudaram um atributo específico da QI: a confiabilidade da informação. Por outro lado, Paim et al. (1996) estudaram o problema de definição da QI.

Contudo, os estudos citados não se focalizam em métodos de avaliação da QI. Neste sentido, outra linha de pesquisa sobre o assunto tem surgido para operacionalizar esta questão, de maneira que as empresas possam realizar avaliações da sua própria QI. Pode-se citar, por exemplo, 0 trabalho de

Kahn et al. (2002) que propuseram um modelo de análise das dimensões da QI no ambiente de desenvolvimento de produtos e serviços. Este modelo foi aprimorado por Lee et al. (2002) para ser utilizado em um contexto mais geral. Este modelo propõe um questionário de levantamento para identificar a lacuna entre as práticas que facilitam a QI e a situação atual da QI de uma organização.

O modelo proposto por estes autores concentra-se na situação atual das diferentes dimensões da qualidade em uma organização, mas não analisa a situação dos principais fatores que influenciam na mesma. Algo similar ocorre com outro modelo de avaliação, proposto por Michnik e Lo (2009). Estes autores também propuseram um modelo de diagnóstico baseado numa análise hierárquica das dimensões da qualidade. Neste caso os fatores de influência tampouco são considerados. Em ambos os modelos a questão dos atributos e dimensões da QI são o centro da análise.

No campo da QI aplicada especificamente ao ambiente produtivo, podem-se ressaltar os trabalhos desenvolvidos por Favaretto e Vieira (2007) e Favaretto (2007). Favaretto e Vieira (2007) estudaram a QI no planejamento da produção. Estes autores levantaram algumas características da QI em etapas específicas da produção. Posteriormente, Favaretto (2007) propôs uma metodologia 
para a medição da QI neste contexto. Mas o foco destes trabalhos não é aprofundar sobre os fatores de influência que definem a QI no PCP.

Pode-se observar que, nos trabalhos citados, não há uma análise específica dos fatores de influência da QI. A maior parte dos trabalhos focalizaram a atenção nos atributos e dimensões da QI. Trabalhos que estudam alguns fatores de influência da QI, como por exemplo Li e Lin (2006), apresentam estudos de levantamento (survey) e não um método de diagnóstico para as empresas. Além disso, esses trabalhos não têm estudado este tema especificamente na área do PCP. Em razão disso, o presente trabalho propõe estudar quais são as características do ambiente (fatores de influência) que podem prejudicar a QI utilizada no PCP. Além disso, o trabalho apresenta uma estrutura para realizar a análise de diagnóstico dos fatores identificados, de maneira tal que se possa determinar quais deles têm um maior impacto sobre a QI utilizada no PCP. A seguir apresenta-se a metodologia de pesquisa que fundamenta o estudo e, posteriormente, os resultados do trabalho.

\section{MÉTODO DE PESQUISA}

O método seguido nesta pesquisa classifica-se em uma pesquisa aplicada, qualitativa, quantitativa e exploratória. De acordo com os procedimentos utilizados, para a identificação dos fatores de influência na QI, foi utilizada a abordagem de estudo de caso (YIN, 2001). Foi realizado um estudo aprofundado da realidade de uma empresa, sendo utilizados, para isso, diferentes instrumentos de coleta e análise dos dados levantados. A seleção do caso estudado foi por conveniência, considerando que se identificou uma não-conformidade desta empresa em relação à QI fornecida ao PCP. Assim sendo, considera-se um caso relevante de ser analisado para os objetivos do presente trabalho.

Como fontes de evidência do estudo utilizaram-se entrevistas, revisão de documentação e observação-participante. O estudo de caso foi realizado ao longo de um período de seis meses, com visitas semanais à empresa. Para conduzir o mesmo, foram realizadas reuniões de caráter informal com funcionários dos diversos setores da empresa relacionados na pesquisa, visando à obtenção de informações sobre a realidade da mesma sob diferentes pontos de vista, bem como o entendimento das inter-relações entre os processos. Os pesquisadores também tiveram acesso a documentos internos da organização, como planilhas de controle, gráficos e procedimentos de rotina, além de acesso ao sistema ERP, de modo a possibilitar maior contato com as informações que circulam no 
ambiente de trabalho. Após a observação dos pesquisadores, foram realizadas entrevistas semiestruturadas com gestores das principais áreas envolvidas no estudo.

Para o levantamento e classificação dos fatores de influência da QI no PCP utilizou-se uma adaptação da abordagem sociotécnica proposta por Hendrick e Kleiner (2001) para análises macroergonômicos. De acordo com estes autores, os sistemas sociotécnicos podem ser subdivididos em quatro subsistemas que estão inter-relacionados: (i) Subsistema Pessoal, que considera características das equipes, como profissionalismo, características demográficas e aspectos psicossociais; (ii) Subsistema Tecnológico, que considera o ambiente físico e as características do trabalho como equipamentos, ferramentas, automatização, entre outros; (iii) Subsistema Organização do Trabalho, que envolve a forma em que foi projetado o trabalho, grau de centralização e formalização e as práticas utilizadas no trabalho; e (iv) Subsistema do Ambiente Externo, no qual se consideram aspectos externos à empresa, mas que a influenciam, tais como os ambientes socioeconômicos, educação, político, cultural e legal. Todos esses subsistemas sociotécnicos formam parte também do sistema de PCP.

Uma vez classificados os fatores de influência nos quatro subsistemas sociotécnicos, foi realizado um estudo para determinar a importância que esses fatores têm para a QI do PCP da empresa. A estrutura desenvolvida para esta análise baseia-se no método para análise da QI descrito por Gackowski (2005), que se divide em quatro etapas: (i) identificação e priorização dos atributos de qualidade que têm influência nos resultados, classificando-os em primários e secundários; (ii) analises do impacto de cada fator, de acordo com a percepção do usuário; (iii) desenvolvimento de um modelo da situação contendo o que é conhecido, o que não é conhecido, o que deve ser adquirido e um ranking para cada valor de dado/informação e seu respectivo impacto operacional, através de medidas agregadas e (iv) análise de cada valor de dado/ informação com base nos requisitos de qualidade.

Em base às etapas descritas anteriormente, foi desenvolvida uma estrutura de matrizes que permite realizar um diagnóstico da situação da QI no PCP. A estrutura é composta por três matrizes cuja estrutura é similar (conforme a Figura 4), mas com conteúdos de avaliação diferentes. Para essas matrizes, a coluna "subsistemas de influência" corresponde à adaptação da abordagem sociotécnica. A coluna "fatores observados" compreende os $m$ fatores de influência na QI para o PCP, obtidos através do estudo de caso realizado. Os mesmos estão classificados de acordo com seus respectivos subsistemas de influência. As colunas "áreas ou processos de influência" correspondem às $n$ áreas/processos que fornecem informações para o PCP. Cada entrevistado deve 
responder apenas por sua área ou processo. Os preenchimentos $\mathrm{I}_{\mathrm{ij}}, \mathrm{S}_{\mathrm{ij}}$ e $\mathrm{P}_{\mathrm{ij}}$ representam os relacionamentos do fator de influência $i$ com a área funcional $j$ que são usados respectivamente para cada uma das três matrizes de avaliação.

Figura 4 - Estrutura das matrizes de análise

\begin{tabular}{|c|c|c|c|c|c|c|c|}
\hline \multirow[b]{2}{*}{$\begin{array}{l}\text { Subsistemas } \\
\text { de Influência }\end{array}$} & \multirow[b]{2}{*}{$\begin{array}{c}\text { Fatores } \\
\text { Observados }\end{array}$} & \multicolumn{4}{|c|}{ Áreas/Processos de Influência } & \multicolumn{2}{|c|}{ Médias } \\
\hline & & $\begin{array}{c}\text { Setorl } \\
\text { Processo } 1\end{array}$ & $\begin{array}{c}\text { Setorl } \\
\text { Processo } 2\end{array}$ & $\cdots$ & $\begin{array}{c}\text { Setorl } \\
\text { Processo n }\end{array}$ & $\begin{array}{c}\text { Médias } \\
\text { dos } \\
\text { fatores }\end{array}$ & $\begin{array}{c}\text { Médias } \\
\text { dos } \\
\text { Subsist. }\end{array}$ \\
\hline \multirow{3}{*}{ Pessoas } & Fator 1 & $\mathrm{I}_{11} / \mathrm{S}_{11} / \mathrm{P}_{11}$ & $\mathrm{I}_{11} / \mathrm{S}_{11} / \mathrm{P}_{11}$ & $\ldots$ & $\mathrm{I}_{1 \mathrm{n}} / \mathrm{S}_{1 \mathrm{n}} / \mathrm{P}_{1 \mathrm{n}}$ & $\overline{F_{1}}$ & \multirow{3}{*}{$\mathrm{SS}_{1}$} \\
\hline & Fator 2 & $\mathrm{I}_{21} / \mathrm{S}_{21} / \mathrm{P}_{21}$ & $\mathrm{I}_{22} / \mathrm{S}_{22} / \mathrm{P}_{22}$ & $\ldots$ & $\mathrm{I}_{2 \mathrm{n}} / \mathrm{S}_{2 \mathrm{n}} / \mathrm{P}_{2 \mathrm{n}}$ & $\mathrm{F}_{2}$ & \\
\hline & $\ldots$ & $\ldots$ & $\ldots$ & $\ldots$ & $\ldots$ & $\ldots$ & \\
\hline \multirow{3}{*}{ Tecnologias } & $\ldots$ & $\ldots$ & $\ldots$ & $\ldots$ & $\ldots$ & $\ldots$ & \multirow{3}{*}{$\mathrm{SS}_{2}$} \\
\hline & $\ldots$ & $\ldots$ & $\ldots$ & $\ldots$ & $\ldots$ & $\ldots$ & \\
\hline & $\ldots$ & $\ldots$ & $\ldots$ & $\ldots$ & $\ldots$ & $\ldots$ & \\
\hline \multirow{3}{*}{$\begin{array}{l}\text { Método de } \\
\text { trabalho }\end{array}$} & $\ldots$ & $\ldots$ & $\ldots$ & $\ldots$ & $\ldots$ & $\ldots$ & \multirow{3}{*}{$\mathrm{SS}_{3}$} \\
\hline & $\ldots$ & $\ldots$ & $\ldots$ & $\ldots$ & $\ldots$ & $\ldots$ & \\
\hline & $\ldots$ & $\ldots$ & $\ldots$ & $\ldots$ & $\ldots$ & $\ldots$ & \\
\hline \multirow{4}{*}{$\begin{array}{l}\text { Ambiente } \\
\text { Externo }\end{array}$} & $\ldots$ & $\ldots$ & $\ldots$ & $\ldots$ & $\ldots$ & $\ldots$ & \multirow{3}{*}{$\mathrm{SS}_{4}$} \\
\hline & $\ldots$ & $\ldots$ & $\ldots$ & $\ldots$ & $\ldots$ & $\ldots$ & \\
\hline & Fator $\mathbf{m}$ & $\mathrm{I}_{\mathrm{m} 1} / \mathrm{S}_{\mathrm{m} 1} / \mathrm{P}_{\mathrm{m} 1}$ & $\ldots$ & $\ldots$ & $\mathrm{I}_{\mathrm{mn}} / \mathrm{S}_{\mathrm{mn}} / \mathrm{P}_{\mathrm{mn}}$ & $\mathrm{F}_{\mathrm{m}}$ & \\
\hline & $\begin{array}{c}\text { Média das } \\
\text { áreas/processos }\end{array}$ & $\mathrm{AP}_{1}$ & $\mathrm{AP}_{2}$ & $\ldots$ & $A P_{n}$ & & \\
\hline
\end{tabular}

A primeira matriz avalia o grau de importância $\left(I_{i j}\right)$ que cada fator de influência $i$ tem para as informações fornecidas ao PCP na área ou processo j. A pergunta correspondente é: Quanto que o fator $i$ é importante para as questões referentes às informações do PCP na área/processo $j$ ? Nesta avaliação utiliza-se uma escala de 0 a 5, na qual 0 significa que o fator não tem importância nessa área e 5 que é muito importante na determinação da QI que receberá o PCP.

Para a segunda matriz, o relacionamento $\left(S_{i j}\right)$ representa a situação atual em que se encontra o fator de influência $i$ na área funcional ou processo $j$. A pergunta correspondente a cada nota é: Qual é o grau de dificuldade que o fator $i$ possui na área/processo $j$ ? Nesta avaliação utiliza-se uma escala de 0 a 5 , na qual 0 representa a ausência de problemas do fator avaliado (situação muito boa) e 5 representa uma situação muito ruim do fator avaliado dentro da área.

Finalmente, realizados os preenchimentos de importância dos fatores nas áreas/processos para a QI do PCP $\left(l_{i j}\right)$ e situação atual dos fatores nessas áreas/processos $\left(S_{\mathrm{ij}}\right)$, procede-se a realizar a priorização final por meio de uma última matriz, cuja estrutura é similar às anteriores. Nesta matriz avalia-se o peso de prioridade de melhoria $\left(\mathrm{P}_{\mathrm{ij}}\right)$ para o fator $i$ na área ou processo $j$. O peso $\mathrm{P}_{\mathrm{ij}}$ é calculado conforme a Equação 1: 


$$
P_{i j}=I_{i j} \times S_{i j}
$$

Onde:

$P_{i j}$ é o peso de prioridade de melhoria para o fator $i$ na área ou processo $j$;

$I_{i j}$ é o grau de importância do fator $i$ na área ou processo $j$;

$S_{i j}$ é a situação atual do fator $i$ na área ou processo $j$;

Os resultados do $\mathrm{P}_{\mathrm{ij}}$ podem variar de 1 (o fator não apresenta nenhuma importância e satisfaz plenamente às expectativas) a 25 (o fator apresenta alta importância e não satisfaz às expectativas). Esta priorização final indica o potencial de melhoria dos fatores em cada área.

Além disso, nas matrizes, também são calculadas as médias de cada fator ${ }_{i}\left(F_{i}\right)$, as médias dos subsistemas $\left(\mathrm{SS}_{\mathrm{k}}\right)$ e as médias de cada área/processo $j\left(\mathrm{AP}_{\mathrm{j}}\right)$, conforme as Equações 2,3 e 4 respectivamente.

$$
F_{i}=\frac{\sum_{j=1}^{n} R_{i j}}{n}
$$

Onde:

$F_{i j}$ é a média simples das avaliações dos fatores;

$P_{i j}$ é o peso de prioridade de melhoria para o fator $i$ na área ou processo $j$;

$n$ é a quantidade de áreas/processos que influenciam na QI para o PCP.

$$
S S_{k}=\frac{\sum_{j=1}^{n} \sum_{i=1}^{l} P_{i j}}{n}
$$

Onde:

$S S_{k}$ é a média simples das avaliações dos subsistemas de influência;

$P_{i j}$ é o peso de prioridade de melhoria para o fator $i$ na área ou processo $j$;

k é o número do subsistema analisado (1 a 4);

I é a quantidade de fatores $i$ que correspondem ao subsistema k;

$n$ é a quantidade de áreas/processos que influenciam na QI para o PCP; 


$$
A_{j}=\frac{\sum_{i=1}^{m} p_{i j}}{m}
$$

Onde:
$A_{i j}$ é a média simples das avaliações das áreas/processos;
$P_{i j}$ é o peso de prioridade de melhoria para o fator $i$ na área ou processo $j$;
$m$ é a quantidade de fatores que influenciam na QI para o PCP.

As médias dessas equações indicam os fatores (Eq.2), subsistemas (Eq.3) mais afetados e as áreas funcionais mais comprometidas (Eq.4) na situação atual da QI para o PCP. A seguir são apresentados os resultados de uma aplicação prática do método de análise proposto.

\section{RESULTADOS DO TRABALHO}

O estudo de caso foi realizado em uma empresa de médio porte que desenvolve e fabrica equipamentos sob encomenda para movimentação e preparo de granéis sólidos, situada na região sul do Brasil. A empresa possui uma equipe de engenharia, uma unidade de manufatura e uma equipe de montagem externa, além dos setores de suporte. O sistema de produção segue a lógica engineering-to-order, uma vez que cada produto é desenvolvido e fabricado de forma customizada para atender às necessidades de cada cliente. Após o contato inicial do setor comercial da empresa com o cliente, é realizada a identificação das demandas deste, e um projeto específico é desenvolvido pela equipe de engenharia. O produto final é composto por centenas de conjuntos, subconjuntos e componentes. As especificações de produção de cada conjunto são enviadas pela engenharia ao setor de PCP. As ordens de produção são então criadas e enviadas para a produção conforme sistemática de programação do setor de PCP.

O processo de PCP é realizado por uma área dedicada especificamente a este fim, que é composta por um coordenador e uma equipe de oito funcionários. As informações recebidas pelo setor de PCP são classificadas em dois tipos: informações de demanda e informações de capacidade. O primeiro grupo de informações chega ao PCP através dos seguintes setores: Comercial, Administração de Contratos, Engenharia e Padronização. O segundo grupo de informações é fornecido pelos setores de Produção, Montagem Externa, Métodos e Processos e Suprimentos. Na Figura 5, são descritas as funções das áreas relacionadas com PCP que fornecem informação para o mesmo. 
Figura 5 - Descrição das áreas da empresa que fornecem informações ao PCP

\begin{tabular}{|l|l|}
\hline \multicolumn{1}{|c|}{$\begin{array}{c}\text { Área da empresa que } \\
\text { fornece } \\
\text { informações para o PCP }\end{array}$} & \multicolumn{1}{c|}{ Principal função da área } \\
\hline Administração de contratos & $\begin{array}{l}\text { Gerencia os contratos em andamento na empresa, realizando o } \\
\text { contato direto com os clientes }\end{array}$ \\
\hline Comercial & Dedica-se à busca de novos clientes \\
\hline Engenharia & Encarregada do processo de desenvolvimento dos produtos \\
\hline Padronização & Realiza o cadastro de novas estruturas no sistema \\
\hline Produção & Encarregada da fabricação dos produtos \\
\hline Montagem externa & Realiza a montagem dos produtos nas instalações dos clientes \\
\hline Métodos e processos & Realiza o controle e as melhorias nos processos de fabricação \\
\hline Suprimentos & $\begin{array}{l}\text { Realiza a compra e armazenagem das matérias-primas e } \\
\text { componentes de montagem }\end{array}$ \\
\hline
\end{tabular}

A existência de problemas no PCP da empresa, tais como: mudanças frequentes de planejamento, atrasos de entrega, falta de informações de capacidade e status produtivo, foi o motivou o estudo, visando determinar as causas relacionadas a esses problemas de falta de QI para o PCP. A seguir apresentam-se os fatores que influenciam à QI identificados no caso estudado.

\subsection{Análise dos Fatores Sociotécnicos que afetam a QI no PCP}

Através da análise sociotécnica, os fatores de influência na QI para o PCP foram classificados nos quatro subsistemas detalhados a seguir.

\subsubsection{Subsistema Pessoal}

O primeiro subsistema de influência se refere a fatores relacionados às pessoas. Nele, foram observados os seguintes fatores de influência na QI.

i. Motivação / interesse: considera a falta de motivação dos funcionários para compartilhar informações. No estudo, foi possível observar que isto ocorre devido principalmente à falta de conscientização das pessoas quanto à importância de suas tarefas para a empresa, bem como e pouco envolvimento das mesmas na definição dos objetivos estratégicos da organização, o que acarreta em um indevido tratamento de informações relevantes para a área de PCP.

ii. Carregamento de dados: em alguns casos podem existir erros humanos no carregamento de dados no sistema, que resulta em informações errôneas ou inclusive perda de informações. 
iii. Competências técnicas: funcionários com menor formação técnica ou sem treinamento para a realização de suas tarefas possuem maior dificuldade para o tratamento das informações que recebem de outras áreas, tendo dificuldades para identificar as informações relevantes para a tomada de decisões.

iv. Comportamento colaborativo: a falta de comportamento colaborativo entre as pessoas, dentro e entre os setores, cria um ambiente que dificulta a troca de informações e restringe os esforços para melhoria da QI transmitida.

v. Comunicação: a falta de comunicação entre as pessoas, dentro e entre os setores, gera um distanciamento entre os fornecedores e os usuários da informação, e isto prejudica a qualidade da mesma.

vi. Registros formais: para algumas operações, não existem procedimentos formalizados. Nesses casos, a realização de atividades informais, sem padronização e registros, cria um potencial para a perda ou distorção de informações ao longo do processo.

\subsubsection{Subsistema Tecnológico}

O segundo subsistema de influência analisado é o referente às Tecnologias utilizadas na empresa para apoio às informações. Neste subsistema foram observados os seguintes fatores:

i. Abrangência do Sistema: sistemas de armazenamento, tratamento de dados e informações cujas demandas do processo não são supridas resultam em informações perdidas e surgimento de controles paralelos cuja ambiguidade gerada, além de ineficiente, prejudica a tomada de decisões.

ii. Confiabilidade do Sistema: a incerteza sobre os históricos de dados existentes e a falta de rastreabilidade desses dados geram dúvidas sobre as decisões a serem tomadas no futuro.

iii. Praticidade de uso: elementos do sistema que são pouco práticos, complexos, lentos ou burocráticos acabam por serem deixados de lado no meio da rotina dos trabalhadores e, dessa forma, informações são perdidas ou simplesmente desconsideradas.

iv. Automação: o baixo nível de automação dos processos, exigindo muitas tarefas manuais, favorece a perda de informações bem como a transmissão de informações de qualidade comprometida. 


\subsubsection{Subsistema Organização do Trabalho}

O terceiro subsistema de influência na QI faz referência à organização do trabalho e aos métodos utilizados para a mesma, sobre os quais se observou os seguintes fatores:

i. Planejamento do trabalho: a falta de planejamento do trabalho cria incongruências entre as informações do planejamento e a execução, além de prejudicar a confiabilidade de informações estabelecidas no planejamento. Isto também gera elevada variabilidade nos processos dificultando a obtenção e transmissão de informações confiáveis.

ii. Explicitação de práticas / responsabilidades: a falta de definições dentro dos processos, através de documentos e práticas que estabeleçam e esclareçam o que a empresa espera de cada funcionário em cada atividade prejudica a QI à medida que informações deixam de ser transmitidas pelo fato de ninguém ser formalmente responsável por isto.

iii. Processos de controle: processos de controle incompletos, mal estruturados e sem padronização geram tratamento de dados ineficientes. As informações geradas por eles podem ser pouco confiáveis.

iv. Sistemática de coleta de dados: a definição de procedimentos eficientes utilizados para a coleta de dados na empresa é um elemento-chave para a QI. Em vários casos, observa-se que a obtenção e carregamento de dados errôneos não é devido apenas ao erro humano, mas principalmente aos procedimentos equivocados para coletá-los.

v. Estrutura organizacional por processo: em estruturas departamentalizadas, nas quais cada atividade é analisada separadamente na empresa, perde-se a noção da importância que determinadas informações podem ter nos processos seguintes. Isto leva a tratamentos grosseiros de informações em áreas onde as mesmas têm pouca influência, bem como má transmissão dessas informações para os setores onde elas têm maior relevância, prejudicando o processo como um todo.

\subsubsection{Subsistema Ambiente Externo}

O quarto subsistema analisado trata dos fatores de influência na QI relacionados ao ambiente externo à empresa, incluindo fatores que pertencem ao entorno na qual esta se encontra inserida. A seguir descrevem-se os mesmos: 
i. Conhecimento do comportamento do mercado: características como instabilidade no mercado, crises econômicas, mudanças políticas, relações de concorrência, alterações de demanda, etc., afetam a QI enviada ao PCP. Isto se deve principalmente à dificuldade de captar informações confiáveis no mercado, o que também acaba dificultando o planejamento da produção e, portanto, acaba-se priorizando as decisões de curto prazo.

ii. Comunicação com clientes: se a comunicação da empresa com seus clientes é incompleta, erros e mudanças nos projetos se repetem com maior frequência.

iii. Comunicação com fornecedores: a dificuldade de criar níveis apropriados de integração com os fornecedores, para utilizar informações dos mesmos no planejamento das vendas e produção, assim como a falta de informações confiáveis dos mesmos, prejudica a Q। enviada ao PCP.

\subsection{Análise dos fatores em relação às fontes de informação para o PCP}

Definidos e conhecidos os fatores de influência, obtiveram-se ponderações numéricas da importância de cada fator de influência para a QI enviada ao PCP em cada uma das áreas funcionais analisadas. A importância de cada fator está diretamente relacionada ao quanto o determinado fator influencia na QI. A tabela com os resultados é apresentada na Figura 6. 
Figura 6 - Importância dos fatores de influência para cada área/processo da empresa

\begin{tabular}{|c|c|c|c|c|c|c|c|c|c|c|c|}
\hline & & \multicolumn{8}{|c|}{ Áreas de Influência sobre a QI para o PCP } & \multirow[b]{2}{*}{$\begin{array}{l}\frac{1}{0} \\
\frac{7}{\pi} \\
\frac{0}{0} \\
\frac{\pi}{2} \\
\stackrel{0}{ \pm}\end{array}$} & \multirow[b]{2}{*}{ 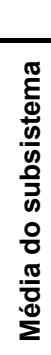 } \\
\hline $\begin{array}{l}\text { Subsistemas } \\
\text { de Influência }\end{array}$ & Fatores Observados & 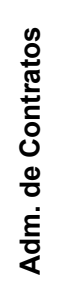 & 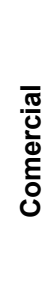 & 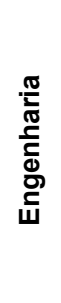 & 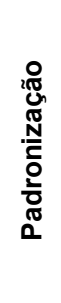 & 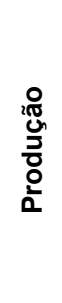 & 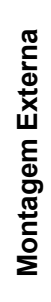 & 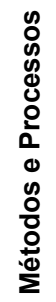 & 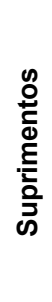 & & \\
\hline \multirow{6}{*}{ Pessoas } & Motivação / Interesse & 4 & 4 & 4 & 3 & 5 & 4 & 4 & 4 & 4,0 & \multirow{6}{*}{4,3} \\
\hline & Carregamento de dados & 5 & 4 & 5 & 4 & 4 & 5 & 5 & 5 & 4,6 & \\
\hline & Competências técnicas & 4 & 4 & 5 & 4 & 4 & 4 & 3 & 4 & 4,0 & \\
\hline & Comportamento colaborativo & 4 & 4 & 4 & 4 & 5 & 4 & 3 & 4 & 4,0 & \\
\hline & Comunicação & 4 & 4 & 5 & 4 & 4 & 5 & 4 & 5 & 4,4 & \\
\hline & Registros formais & 5 & 4 & 5 & 4 & 4 & 5 & 5 & 5 & 4,6 & \\
\hline \multirow{4}{*}{ Tecnologias } & Abrangência & 5 & 3 & 5 & 3 & 4 & 4 & 5 & 5 & 4,3 & \multirow{4}{*}{4,2} \\
\hline & Confiabilidade & 5 & 3 & 5 & 4 & 5 & 5 & 5 & 5 & 4,6 & \\
\hline & Praticidade de uso & 4 & 3 & 4 & 3 & 5 & 4 & 5 & 4 & 4,0 & \\
\hline & Automação & 4 & 3 & 4 & 4 & 4 & 4 & 4 & 4 & 3,9 & \\
\hline \multirow{5}{*}{$\begin{array}{c}\text { Método de } \\
\text { Trabalho }\end{array}$} & Planejamento do trabalho & 5 & 3 & 4 & 3 & 5 & 5 & 4 & 5 & 4,3 & \multirow{5}{*}{4,0} \\
\hline & $\begin{array}{l}\text { Explicitação de práticas / } \\
\text { responsabilidades }\end{array}$ & 3 & 3 & 4 & 3 & 4 & 4 & 4 & 4 & 3,6 & \\
\hline & Processos de controle & 3 & 3 & 4 & 4 & 4 & 4 & 4 & 5 & 3,9 & \\
\hline & Sistemática de coleta de dados & 5 & 3 & 4 & 3 & 4 & 4 & 5 & 5 & 4,1 & \\
\hline & Estrutura organizacional por processo & 3 & 3 & 4 & 5 & 5 & 4 & 3 & 4 & 3,9 & \\
\hline \multirow{4}{*}{$\begin{array}{c}\text { Ambiente } \\
\text { Externo }\end{array}$} & $\begin{array}{l}\text { Conhecimento do comportamento do } \\
\text { mercado }\end{array}$ & 3 & 3 & 3 & 1 & 3 & 4 & 4 & 4 & 3,1 & \multirow{3}{*}{3,5} \\
\hline & Comunicação com clientes & 4 & 3 & 5 & 1 & 5 & 5 & 4 & 5 & 4,0 & \\
\hline & Comunicação com fornecedores & 2 & 3 & 5 & 1 & 5 & 4 & 3 & 5 & 3,5 & \\
\hline & Média da área & 4,0 & 3,3 & 4,4 & 3,2 & 4,4 & 4,3 & 4,1 & 4,6 & & \\
\hline
\end{tabular}

$\mathrm{Na}$ matriz da Figura 6 observa-se que os subsistemas Pessoas e Tecnologias são os considerados mais importantes para a QI fornecida ao PCP, tendo além das médias mais altas, os fatores com maiores médias: Carregamento de dados e Registros formais, para o primeiro; e Confiabilidade para o segundo. Observa-se também que as áreas Suprimentos, Engenharia e Produção respectivamente são as de maior importância para a QI no PCP do caso estudado.

A segunda matriz (Figura 7) foi utilizada para obter as avaliações numéricas quanto à situação atual de cada fator de influência em cada área funcional analisada. A situação atual de cada fator representa o quanto que este fator satisfaz as expectativas em relação ao nível considerado ótimo para a empresa. Nesta matriz notas baixas representam ausência de problemas e notas altas destacam que o fator é problemático na área ou processo avaliado. 
Figura 7 - Situação atual dos fatores de influência para cada área/processo da empresa

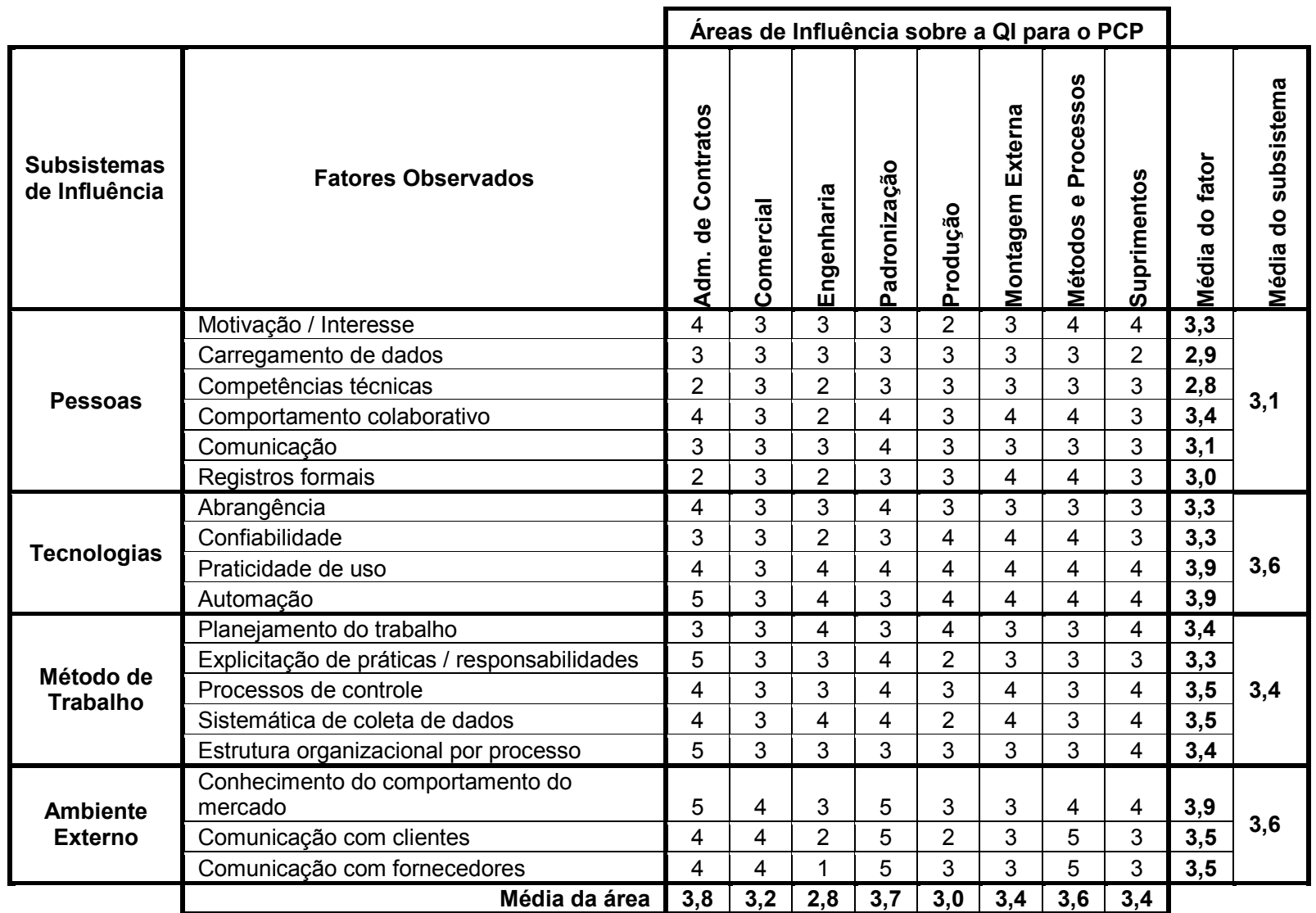

A partir da matriz da Figura 7, pode-se observar que os subsistemas Tecnologias e Ambiente Externo são os que maiores problemas têm em relação à QI fornecida ao PCP. Os fatores que apresentam maiores problemas são: Praticidade de uso; Automação e Conhecimento do comportamento do mercado. Em relação às áreas e processos, as que apresentam maiores problemas para fornecer informações de qualidade para o PCP são: Administração de Contratos e Padronização. Obtidas as avaliações numéricas a respeito da importância e situação atual dos fatores de influência, realiza-se o produto dos resultados das duas matrizes anteriores (Figura 6 e 7) na matriz que é apresentada na Figura 8. 
Figura 8 - Potencial de melhoria dos fatores de influência para cada área/processo da empresa

\begin{tabular}{|c|c|c|c|c|c|c|c|c|c|c|c|}
\hline \multirow[b]{2}{*}{$\begin{array}{l}\text { Subsistemas } \\
\text { de Influência }\end{array}$} & \multirow[b]{2}{*}{ Fatores Observados } & \multicolumn{8}{|c|}{ Áreas de Influência sobre a QI para o PCP } & \multirow[b]{2}{*}{ 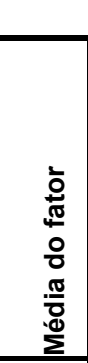 } & \multirow[b]{2}{*}{ 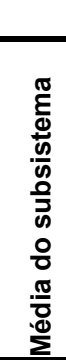 } \\
\hline & & 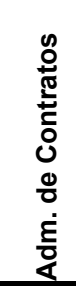 & 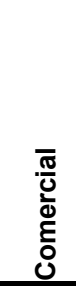 & 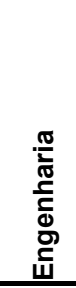 & 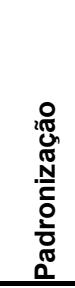 & 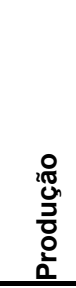 & 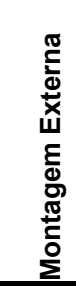 & 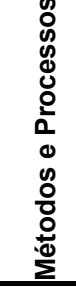 & 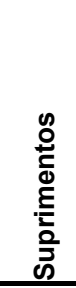 & & \\
\hline \multirow{6}{*}{ Pessoas } & Motivação / Interesse & 16 & 12 & 12 & 9 & 10 & 12 & 16 & 16 & 12,9 & \multirow{6}{*}{13,0} \\
\hline & Carregamento de dados & 15 & 12 & 15 & 12 & 12 & 15 & 15 & 10 & 13,3 & \\
\hline & Competências técnicas & 8 & 12 & 10 & 12 & 12 & 12 & 9 & 12 & 10,9 & \\
\hline & Comportamento colaborativo & 16 & 12 & 8 & 16 & 15 & 16 & 12 & 12 & 13,4 & \\
\hline & Comunicação & 12 & 12 & 15 & 16 & 12 & 15 & 12 & 15 & 13,6 & \\
\hline & Registros formais & 10 & 12 & 10 & 12 & 12 & 20 & 20 & 15 & 13,9 & \\
\hline \multirow{4}{*}{ Tecnologias } & Abrangência & 20 & 9 & 15 & 12 & 12 & 12 & 15 & 15 & 13,8 & \multirow{4}{*}{14,9} \\
\hline & Confiabilidade & 15 & 9 & 10 & 12 & 20 & 20 & 20 & 15 & 15,1 & \\
\hline & Praticidade de uso & 16 & 9 & 16 & 12 & 20 & 16 & 20 & 16 & 15,6 & \\
\hline & Automação & 20 & 9 & 16 & 12 & 16 & 16 & 16 & 16 & 15,1 & \\
\hline \multirow{5}{*}{$\begin{array}{l}\text { Método de } \\
\text { Trabalho }\end{array}$} & Planejamento do trabalho & 15 & 9 & 16 & 9 & 20 & 15 & 12 & 20 & 14,5 & \multirow{5}{*}{13,4} \\
\hline & Explicitação de práticas / respons. & 15 & 9 & 12 & 12 & 8 & 12 & 12 & 12 & 11,5 & \\
\hline & Processos de controle & 12 & 9 & 12 & 16 & 12 & 16 & 12 & 20 & 13,6 & \\
\hline & Sistemática de coleta de dados & 20 & 9 & 16 & 12 & 8 & 16 & 15 & 20 & 14,5 & \\
\hline & Estrutura organiz. por processo & 15 & 9 & 12 & 15 & 15 & 12 & 9 & 16 & 12,9 & \\
\hline \multirow{4}{*}{$\begin{array}{c}\text { Ambiente } \\
\text { Externo }\end{array}$} & Conhecim.do comp.do mercado & 15 & 12 & 9 & 5 & 9 & 12 & 16 & 16 & 11,8 & \multirow{3}{*}{$11, \varepsilon$} \\
\hline & Comunicação com clientes & 16 & 12 & 10 & 5 & 10 & 15 & 20 & 15 & 12,9 & \\
\hline & Comunicação com fornecedores & 8 & 12 & 5 & 5 & 15 & 12 & 15 & 15 & 10,9 & \\
\hline & Média da área & 14,7 & 10,5 & 12,2 & 11,3 & 13,2 & 14,7 & 14,8 & 15,3 & & \\
\hline
\end{tabular}

Observando-se os resultados finais da Figura 8, pode-se encontrar o potencial de melhoria de cada fator $i$ para o setor $j$ na empresa estudada. Na Figura 9 apresentam-se as prioridades no potencial de melhoria de QI no PCP em relação aos subsistemas sociotécnicos. Nesta figura podese observar que no caso estudado as tecnologias oferecem o maior potencial de melhoria na QI para o PCP. Por outro lado, na Figura 10 apresentam-se os resultados discriminados por fatores de influência, nos quais se observa que os três principais são a Praticidade do Uso, Automação e Confiabilidade.

Figura 9 - Prioridade no potencial de melhoria dos subsistemas de influência

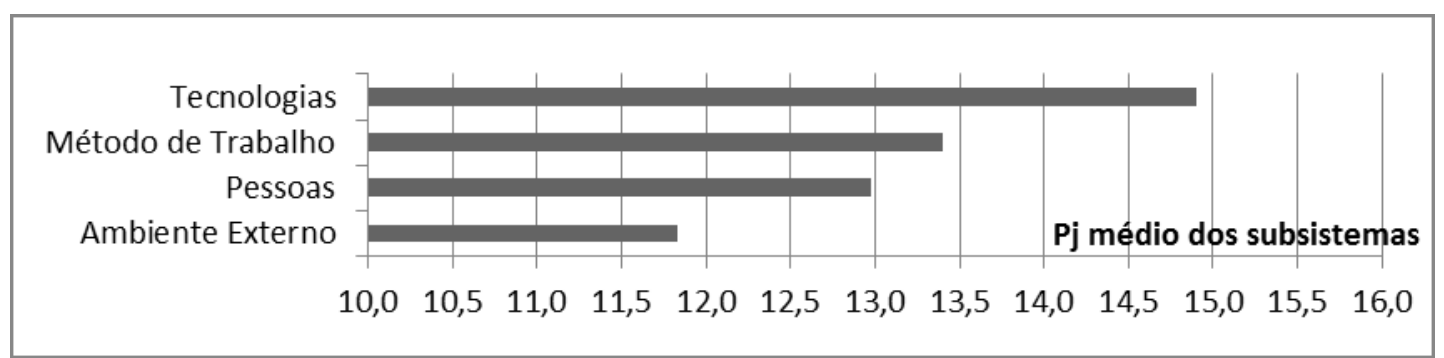

Revista Produção Online, Florianópolis, SC, v.13, n. 1, p. 37-60, jan./mar. 2013. 
Figura 10 - Potencial de melhoria dos fatores de influência na QI para o PCP

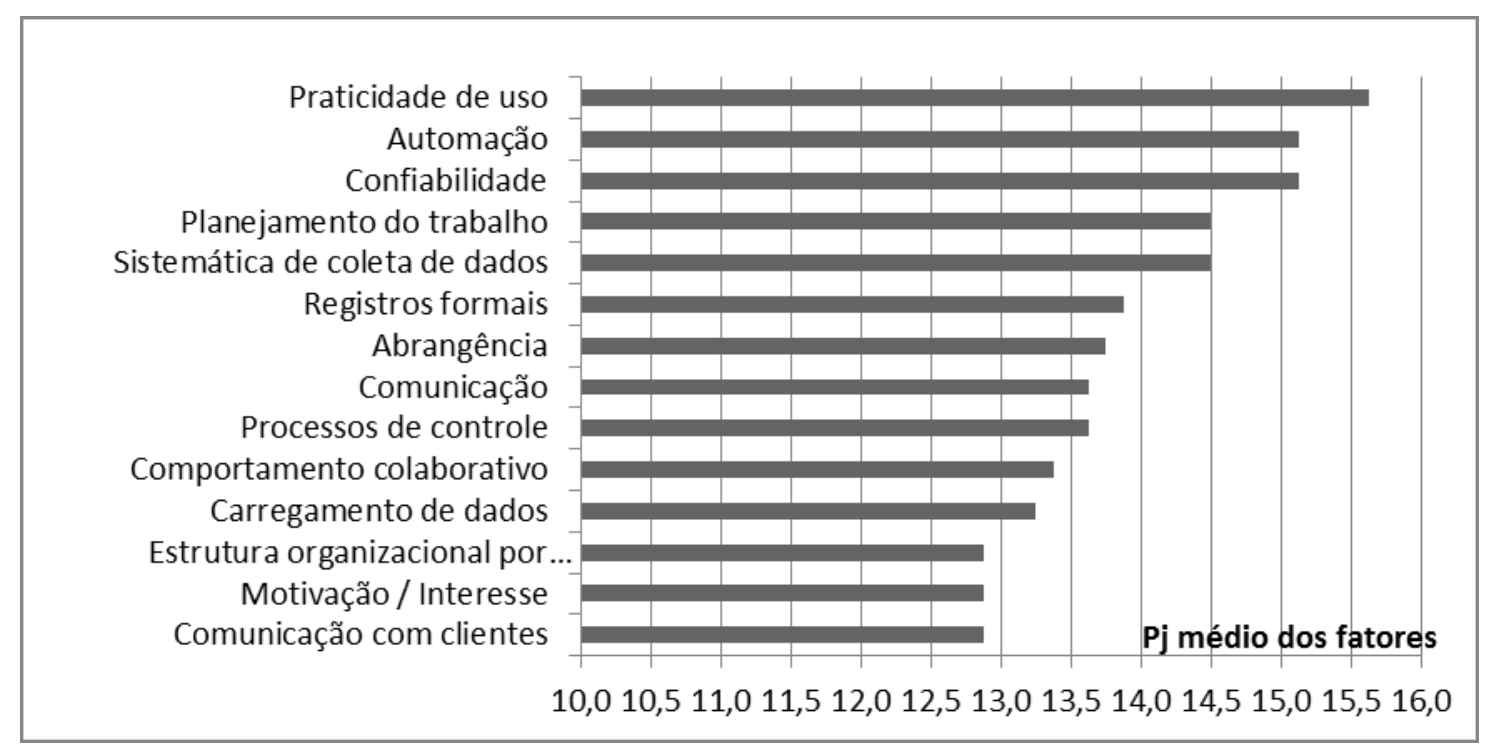

A partir de análise da Figura 10, pode-se observar a praticidade de uso das tecnologias disponíveis na empresa como o fator com maior potencial de melhoria. Tal fato deve-se à empresa utilizar um sistema ERP projetado para empresas com sistemas de produção voltados para produtos padronizados. Essa falta de flexibilidade dificulta as inovações e alterações inerentes aos sistemas de produção do tipo engineering-to-order, como é o caso da empresa estudada. Além disso, a produção de um mix de produtos bastante variado é dificultada por restrições de tecnologias não projetadas para tal, sendo também uma das causas da dificuldade para a QI. Nesse sentido, também se destaca a automação, pois este fator apresenta problemas devido à alta variabilidade dentro dos processos da empresa que dificultam a mesma. Segundo os entrevistados, a falta de confiabilidade nas tecnologias utilizadas ocorre em função do pouco investimento em TI realizado pela empresa, acarretando na obsolescência de equipamentos e softwares utilizados. O quarto fator de maior potencial de melhoria, planejamento do trabalho, está relacionado a uma dificuldade observada na empresa de se adequar às mudanças que ocorrem ao longo do processo de forma flexível. Pode-se constatar que um melhor planejamento das atividades diminuiria a ocorrência das alterações nos projetos que prejudicam a QI enviada ao PCP.

$\mathrm{Na}$ Figura 11, é apresentado um gráfico que classifica as áreas funcionais de forma decrescente também em relação ao potencial de melhoria. Nesta figura, observa-se que a área de Suprimentos é a de maior potencial de melhorias, seguida pelas áreas de Métodos e Processos, Montagem Externa e Administração de Contratos. Os pontos de melhoria para a QI no PCP dentro de cada uma dessas áreas podem ser identificados na matriz da Figura 8, nas respectivas colunas 
para cada área/processo, onde se podem observar quais os fatores da QI, numa área ou processo específico, que têm maior potencial de melhoria. Assim sendo, por exemplo, para a área de Suprimentos, os fatores mais relevantes são: Planejamento do trabalho, Processos de controle, Sistemática de coleta de dados e Estrutura organizacional. Contudo, como se observou na Figura 10, estes fatores não são os que têm maior impacto global nos processos da empresa.

Figura 11 - Potencial de melhoria das áreas de influência

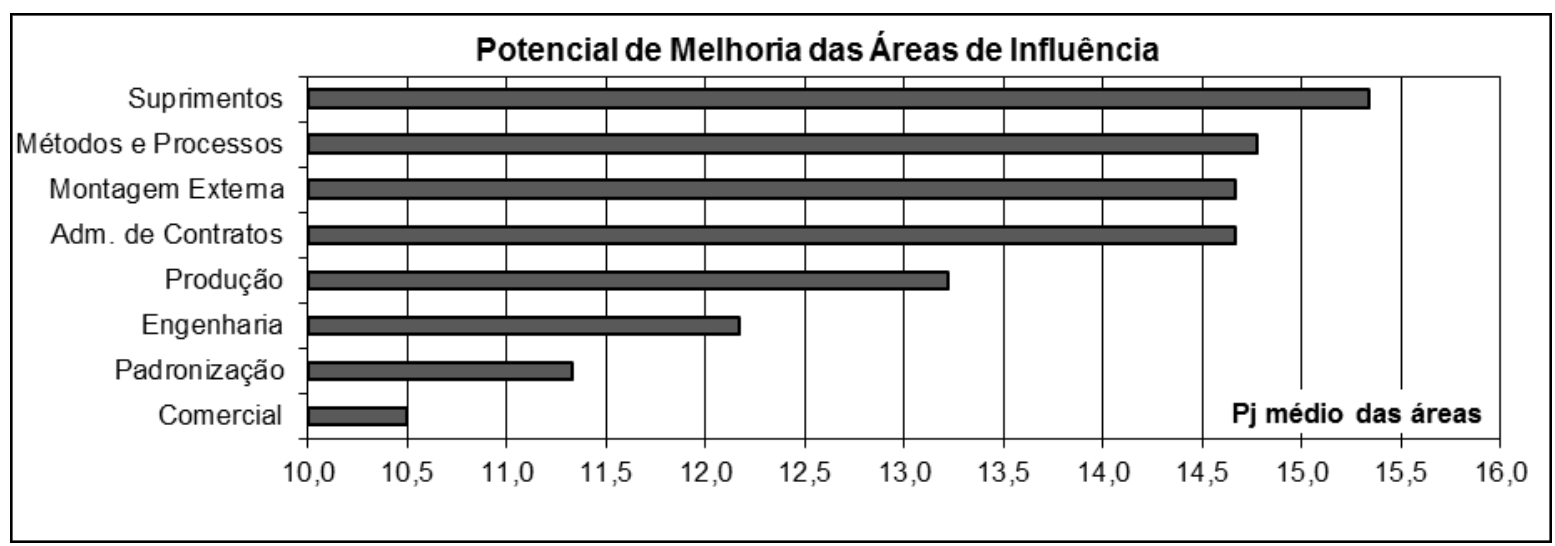

\section{CONSIDERAÇÕES FINAIS}

Este trabalho apresentou uma pesquisa a respeito de fatores de influência na qualidade de informação (QI) para o PCP. Para isto, foi realizado um estudo de caso, a fim de identificar os principais fatores de influência na QI para o PCP em uma empresa metal-mecânica que opera na modalidade engineering-to-order. Para a classificação dos fatores de influência utilizou-se a abordagem sociotécnica. Os fatores identificados foram priorizados através de uma matriz de relacionamentos também proposta neste trabalho. Como principais resultados identificaram-se quatro categorias de fatores de influência: (i) Pessoas; (ii) Tecnologias; (iii) Método de Trabalho e (iv) Ambiente Externo. Também se identificaram um total de 18 fatores distribuídos nessas categorias.

Foi desenvolvido um conjunto de matrizes para realizar o diagnóstico da situação da QI no PCP da empresa baseada nos fatores levantados. Após da análise de relacionamento dos fatores de influência identificaram-se quatro elementos prioritários a serem melhorados no caso estudado: Praticidade de uso, Automação, Confiabilidade e Planejamento do trabalho; sendo os três primeiros relacionados às Tecnologias e o último ao Método de trabalho. Todos estes fatores influenciam na QI para o PCP. Também se identificou que os problemas dos mesmos são provenientes principalmente das áreas comercial, contratos e produção. 
As principais contribuições dos resultados apresentados neste trabalho são duas. Por um lado, o método de diagnóstico utilizado pode ser aplicado em outras empresas para identificar seus problemas em relação à QI para o PCP. Este método é baseado em um enfoque sociotécnico dos fatores de influência na QI. O método realiza a priorização dos fatores de influência na QI para o PCP e das principais áreas onde existem problemas na QI para o PCP e que, portanto, podem ser melhoradas.

Os resultados práticos obtidos ajudam a ampliar o conhecimento em relação aos possíveis problemas que podem ser apresentados na QI para o PCP. Futuros trabalhos mais abrangentes deveriam ser realizados para identificar fatores gerais de influência em um contexto industrial mais amplo. Além disso, algumas limitações do trabalho deixam a possibilidade de serem realizadas futuras pesquisas sobre o tema. Uma das limitações do presente trabalho é o fato de os Fatores de Influência na QI terem sido considerados como um conjunto geral de características que tornam a informação apropriada ou não para o PCP. Contudo, futuros trabalho deveriam tentar discriminar todos os elementos que definem a QI, para assim conseguir identificar, além das causas que prejudicam a QI levantadas neste trabalho, também em quais características da QI estas causas influenciam. Isto significa que, por exemplo, enquanto que alguns fatores podem prejudicar a velocidade em que a informação é transmitida dentro da organização, outros podem prejudicar não a velocidade, mas o conteúdo dessa informação. Este desdobramento maior das influências é um tópico pertinente para futuras pesquisas.

\section{REFERÊNCIAS}

CIURANA, J.; GARCIA-ROMEU, M.L.; FERRER, I.; CASADESÚS, M. A model for integrating process planning and production planning and control in machining processes. Robotics and Computer-Integrated Manufacturing, v.24, n.4, p.532-544, 2008.

DAVENPORT, T. H.; PRUSAK, L. Conhecimento empresarial. Rio de Janeiro: Campus, 1998.

DUTRA, F.A.F.; ERDMANN, R.H. Uma nova abordagem para o estudo do planejamento e controle da produção (PCP): a ótica da Teoria da Complexidade. In: SIMPÓSIO DE ENGENHARIA DE PRODUÇÃO (SIMPEP), 12., 2006, Bauru, São Paulo. Anais... 2006.

EPPLER, M. Managing information quality: Increasing the value of information in knowledge-intensive products and processes. Berlin, Germany: Springer-Verlag, 2003.

FAVARETTO, F; VIEIRA, G. E. Estudo descritivo da qualidade da informação no planejamento da produção.

Revista Gestão Industrial, 2007. 
FAVARETTO, F. Experimento para análise da implantação da medição da qualidade da informação.

Produção, v.17, n.1, p.151-162, 2007.

GARVIN, D.A. What Does "Product Quality" Really Mean? Sloan Management Review, v. 25, n.1, p.25-43, 1984.

GE, M.; HELFERT, M. A Review of Information quality research. in: international conference on information quality (ICIQ-07), 12., 2007, MIT, Massachusset. Proceedings... [S.I.: S.n.].

GACKOWSKI, Z.J. Operations quality of data and information: teleological operations research-bases approach, call for discussion. In: INTERNATIONAL CONFERENCE ON INFORMATION QUALITY (ICIQ-07), 12., 2007, MIT, Massachusset. Proceedings... 2007.

HENDRICK, H.W; B.M. KLEINER. Macroergonomics: an introduction to work system design. Santa Monica, CA: Human Factors and Ergonomics Society, 2001.

HUANG, K.T.; LEE, Y.W.; WANG, R.Y. Quality information and knowledge. New Jersey: Prentice-Hall, 1999.

KAHN, B. K.; STRONG, D. M.; WANG, R. Y. Information quality benchmarks: product and service performance. Communications of the ACM, v.45, n.4, p.84-192, 2002.

KLIPPEL, M.; ANTUNES, J. A. V.; PELLEGRIN, I. Os circuitos do planejamento, programação e controle da produção e dos materiais para indústrias do tipo engineering-to-order - pedidos contra projetos de engenharia. In: ENCONTRO NACIONAL DE ENGENHARIA DE PRODUÇÃO (ENEGEP), 12., 2005, Porto Alegre, RS. Anais... [S.I.: S.n.].

LAUDON, K. C.; LAUDON, J. P. Sistemas de informações gerenciais. São Paulo: Prentice Hall, 2004.

LEE, Y.W; STRONG, D.M.; KAHN, B.; WANG, R.Y. AIMQ: a methodology for information quality assessment. Information \& management, v.40, p.133-146, 2002.

LI, S.; LIN, B. Accessing information sharing and information quality in supply chain management. Decision Support System, v.42, p.1641-1656, 2006.

MARCHAND, D. Managing information quality. In: WORMELL, I. (Ed.). Information quality definitions and dimensions. Proceedings of the NORDINFO Seminar, Royal School of Librarianship. Copenhagen: Taylor Graham, 1989. p.7-17.

MICHNIK, J.; LO, M-C. The assessment of the information quality with the aid of multiple criteria analysis. European Journal of Operation Research, v.195, p.850-856, 2009.

NAUMANN, F.; ROLKER, C. Assessment methods for information quality criteria. Berlin: German research society, 2000.

MORESI, E. A. D. Delineando o valor do sistema de informação de uma organização. Ciência da Informação, v.29, n.1, p.14-24, 2000.

MULA, J.; POLER, R.; GARCÍA-SABATER, J.P; LARIO, F.C.; Models for production planning under uncertainty: A review. International Journal of Production Economics, v.103, n.1, p.271-285, 2006.

OLETO, R.R. Percepção da qualidade da informação. Ciência da Informação, v.35, n.1, p.57-62, 2006. 
PAIM, I.; NEHMY, R. M.; GUIMARÃES, C.. Problematização do conceito qualidade da informação. Perspectivas em Ciência da Informação, v. 1, n. 1, p.111-119, 1996.

REDMAN, T. Data Quality For The Information Age: Norwood: Artech House Publishers, 1996.

SHERMAN, J. D.; BERKOWITZ, D.; SOUDER, W. E. New Product Development Performance and the Interaction of Cross-Functional Integration and Knowledge Management. Journal of Production Innovation Management. v.22, n.5, p.399-411, 2005.

SORDI, J. O.; MEIRELES, M.; GRIJO, R. N. Gestão da qualidade da informação no contexto das organizações: percepções a partir do experimento de análise da confiabilidade dos jornais eletrônicos. Perspectivas em Ciência da Informação, v.13, n.2, p.168-195, 2008.

STVILIA, B.; GASSER, L.; TWIDALE, M. B.; SMITH, L. C.; A Framework for Information Quality Assessment. Journal of the American Society for Information Science and Technology, v.58, n.12, p.1720-1733, 2007.

WAND, Y., WANG, R.Y. Anchoring data quality dimensions in ontological foundations. Communications of the ACM, v.39, n.11, p.86-95, 1996.

WANG, R.Y.; STRONG, D.M. Beyond Accuracy: what data quality means to data consumers. Journal of Management Information Systems, v.12, n.4, p.5-33, 1996.

XU, Lida; WANG, Chengen; LUO, Xiaochuan; SHI, Zhongzhi. Integrating Knowledge Management and ERP in Enterprise Information Systems. Systems Research and Behavioral Science, v.23, n.2, p.147-156, 2006.

YIN, R. K. Estudo de caso: planejamento e métodos. Porto Alegre: Bookman, 2001.

ZACCARELLI, S. B., Programação e controle da produção. 5. ed., São Paulo: Pioneira, 1979. 292 p.

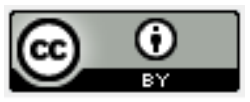

Artigo recebido em 03/06/2011 e aceito para publicação em 01/12/2011. 